\title{
EL PROCESO DE COMUNICACIÓN EN ONCOLOGÍA: RELATO DE UNA EXPERIENCIA
}

\author{
THE COMMUNICATION PROCESS IN ONCOLOGY: REPORTING AN EXPERIENCE
}

\author{
Lourdes Moro Gutiérrez', Elisa Kern de Castro² y Marta González Fernández-Conde ${ }^{3}$ \\ 1 Facultad de Psicología, Universidad de Salamanca, España \\ 2 Universidade do Vale do Rio dos Sinos, Brasil \\ 3 Hospital General de la Santísima Trinidad, Salamanca, España
}

Resumen

Informar a una persona de que tiene cáncer supone un shock para esa persona y para su familia. La enfermedad implica cambios físicos, psíquicos y sociales a los que tienen que aprender a enfrentarse tanto el propio afectado como su entorno más próximo. Conocer que se padece un cáncer aún se asocia inmediatamente con dolor y muerte y esto provoca que el enfermo tenga que enfrentarse a una situación dramática y angustiosa. Para que el afrontamiento de la enfermedad sea lo menos costoso física y emocionalmente para el paciente es necesario que se le atienda desde una perspectiva integral y holista en la que juega un papel destacado en primer lugar, la información que se le aporta sobre el diagnóstico y las posibilidades de tratamiento $y$, en segundo lugar, el proceso de comunicación que se inicia cuando al paciente ya se le ha comunicado el diagnóstico y que se prolonga hasta que se produce la remisión/curación de la enfermedad o el paciente fallece. En este artículo recogemos nuestra experiencia sobre la información y comunicación con pacientes oncológicos en tratamiento de quimioterapia. Es imprescindible trabajar ambos aspectos desde la individualidad de cada caso y para ello proponemos nuestras conclusiones sobre los aspectos básicos en los que es necesario centrarse para conseguir que el enfermo reciba una información adecuada a su caso particular.

Palabras clave: Cáncer, información, comunicación.
Abstract

Telling someone that he/she suffers cancer is a deep shock for the individual and its family as well. The disease involves physical, psychological and social changes that both the patient and his/her social environment have to learn to cope with. Cancer is still readily associated with pain and death, so patients have to face a dramatic and distressing situation. For achieving an emotionally and physically smooth treatment of cancer it is necessary to develop a comprehensive and holistic approach split into three stages. First, information provided about diagnosis and care possibilities. Secondly, the communication process along the treatment period, which may be rather long. Third, and particularly prominent, the communication process during the last stage of the disease, whether it disappears or leads to death. This paper presents our experience on the overall information and communication process with cancer patients who undergo chemotherapy treatment. It is essential to deal with information and communication on a personalized basis for each individual, so our conclusions are focused on the basic topics which have to be addressed for each patient to receive the information the most suitable for his/her particular case.

Key words: Cancer, information, communication.

Correspondencia:

Lourdes Moro Gutiérrez

Facultad de Psicología. Avenida de la Merced, 109-131.37005. Salamanca

E-mail: moro@usal.es 


\section{INTRODUCCIÓN}

Como numerosas estadísticas confirman, la incidencia del cáncer en la población es cada vez mayor. La Sociedad Española de Oncología Médica (SEOM) ${ }^{(1)}$ en España, pronosticaba para el año 2013 una incidencia de nuevos casos de cáncer de entre 103.500 a 104.000 mujeres y de 105.000 a 105.500 hombres. Sin embargo, a pesar de esta alta incidencia y de que esta enfermedad continúa siendo la primera causa de muerte en España, los pronósticos son cada vez mejores debido a que los tratamientos aplicados son mucho más eficaces. Por ello la mortalidad desciende ${ }^{1}$ a la vez que se incrementa el índice de supervivencia ${ }^{2}$, que para algunos tipos de cáncer puede llegar a ser del 82,8\% a los cinco años (AECC) (2). A pesar de estas buenas perspectivas también es cierto que nos encontramos frente a una patología ${ }^{3}$ cuyo diagnóstico continúa provocando miedo e inseguridad. La enfermedad provoca que la persona diagnosticada y su entorno familiar y social tengan que enfrentarse a una situación dramática y angustiosa, que aún en muchas ocasiones se asocia inmediatamente con el dolor y la muerte. La comunicación del diagnóstico de cáncer supone un shock para el paciente y para su familia, que sienten que sus planes de vida quedan radicalmente interrumpidos y el miedo y la inseguridad desde los momentos iniciales de la enfermedad son una constante.

1 A pesar de esta disminución la SEOM estimaba que en el año 2013 morirían por cáncer cerca de 41.000 mujeres y de 62.000 hombres.

2 La página web http://todosomosupervivientes.com estimaba, en su informe sobre "Las necesidades de los supervivientes de cáncer" (2012), que podían ser 1.500.000 las personas supervivientes.

3 Según la AECC la palabra cáncer es un término muy amplio que abarca más de 200 tipos de enfermedades.
La enfermedad implica, en mayor o menor medida, una disminución del bienestar físico, psíquico y social del paciente, lo que a su vez repercute también en su entorno y en las personas que directamente forman parte de él. Todo ello requiere un adecuado tratamiento del enfermo oncológico que implica considerarle una persona conformada por características biopsicosociales y que precisa que se atienda a su salud y a su enfermedad desde una perspectiva integral y holista que supone que no debemos hacer frente a la situación solamente desde un planteamiento biomédico, sino que es necesario también que se atiendan sus necesidades psicológicas y sociales, pues esto va a repercutir en su salud física. Debe trabajarse desde una perspectiva multidisciplinar que permita al paciente conseguir el mayor grado de bienestar posible. No es suficiente con aplicar los ciclos de quimioterapia o las sesiones de radioterapia para responder a sus necesidades.

De cara a conseguir una mejor calidad de vida para el enfermo y para su entorno es necesario que trabajemos en todo el proceso asistencial, desde el momento en que surge la sospecha de que puede tener cáncer, hasta la remisión-curación o el fallecimiento. En este proceso asistencial podemos diferenciar varios momentos fundamentales en los cuales debe insistirse especialmente, tanto desde la investigación como desde la intervención, y que además afectan en diversos ámbitos: el hospital, el domicilio o el trabajo. Durante todo el proceso es clave trabajar los aspectos relacionados con la información sobre el diagnóstico y la comunicación con el enfermo oncológico. Aunque existe un tiempo y un espacio específico en el que se dice al enfermo que tiene cáncer, no podemos reducirlo solo a ese momento temporal y al contexto en el que se produce. Comunicar un diagnóstico de cáncer es un proceso que empieza 
antes de pronunciar la palabra cáncer (o la variante que el médico utilice) y finaliza cuando la persona está en remisión, curación o fallece. La evolución de este proceso está marcada por numerosas variables biológicas (tipo de tumor, localización, etc.), psicológicas (estado de ánimo, funcionamiento familiar, etc.) y sociales (trabajo, apoyo percibido) y repercute no solo en el propio afectado sino también en las personas que componen su entorno más cercano. El diagnóstico implica un importante cambio en la vida del enfermo que da un giro radical ya que pasa a ser una persona "con cáncer", con todo lo que esa etiqueta conlleva. Para todos los enfermos existe un antes y un después del diagnóstico. Su vida y la de las personas más próximas de su entorno cambiará, en mayor o menor medida, pero siempre hay cambios, tanto físicos como emocionales. Su imagen corporal si tiene que someterse a intervenciones quirúrgicas que implican amputaciones, o el posible deterioro físico que pueden padecer debido a los efectos secundarios de los tratamientos, aunque sea temporal, deja una huella en la vida del paciente que cuesta borrar. En el ámbito psicológico, sus emociones, la expresión de sentimientos, los valores o las relaciones familiares y sociales, también sufren modificaciones que afectarán de manera importante a cómo cada uno de ellos entienda y determine su calidad de vida. Uno de los aspectos clave para que el paciente y su familia afronten estos cambios de la manera más adecuada está relacionado con la información y la comunicación que tengan en todo lo que respecta a su enfermedad, tanto en el ámbito físico como emocional.

En este artículo recogemos nuestra experiencia sobre la comunicación con enfermos oncológicos durante el tiempo que están recibiendo tratamiento de quimioterapia. Este trabajo forma parte del Plan de Humanización para pacientes oncoló- gicos en tratamiento de quimioterapia ${ }^{4,5}$ que, desde el año 2007, desarrollamos en el Hospital General de la Santísima Trinidad de Salamanca (España). Nuestro objetivo principal es atender a las necesidades y demandas del enfermo oncológico durante el tiempo que está recibiendo quimioterapia, con el fin de conseguir una mejora en su calidad de vida que también le afecte cuando termine el tratamiento y retome su vida cotidiana.

Todas las personas tienen derecho a recibir información sobre su salud, tal y como se recoge en la Ley 41/2002 sobre "Derechos de información concernientes a la salud y la autonomía del paciente, y la documentación clínica"6. Sin embargo, a pesar del marco legal y de reconocer la importancia de la información en el presente $y$ en el futuro del enfermo, nos encontramos que en la actividad diaria con los afectados continúan existiendo problemas. La mayoría de los trabajos de investigación realizados se centran principalmente en analizar el proceso de informar sobre el diagnóstico y en menor medida en intentar conocer qué es lo que sucede a partir de ese momento. La necesidad de recibir información sobre la enfermedad es una de las demandas más habituales del enfermo y de su entorno y sobre ella y el proceso de comunicación que requiere se centran las siguientes reflexiones.

4 Para ello se firmó un convenio de colaboración entre la Universidad de Salamanca y el citado hospital, siendo las encargadas de su aplicación Lourdes Moro, por parte de la Universidad de Salamanca, y Marta González por parte del Hospital General de la Santísima Trinidad de Salamanca.

5 En la actualidad estamos desarrollando el proyecto de investigación titulado A relaçao profissional da saúde-paciente em oncología: um estudo transcultural Brasil-Espanha, dirigido por la Prof ${ }^{\mathrm{a}}$ Elisa Kern de Castro (Universidade do Vale do Rio dos Sinos-UNISINOS-) y financiado por el CNPq (Brasil).

6 Artículo 5, puntos 1, 3 y 4. 


\section{INFORMACIÓN Y COMUNICACIÓN: ASPECTOS FUNDAMENTALES EN EL PROCESO ONCOLÓGICO}

Aunque en el contexto oncológico información y comunicación se utilizan habitualmente como sinónimos es necesario que diferenciemos ambos términos. De forma general, informar hace referencia al contenido de una comunicación (mensaje), mientras que comunicar se refiere a "cómo el proceso pone en relación dos o más personas-(emisor y receptor- que intercambian informaciones)" (Vidal et al., p. 22) ${ }^{(3)}$. Comunicar es un proceso evolutivo y continuado que facilita "...la comprensión de lo que se entiende por "salud" y cómo se vive; también se facilita la interpretación de las necesidades del paciente y se favorece la comprensión de la intervención profesional". Comunicar significa "informar y facilitar recursos de bienestar; es un itinerario de ayuda, de asistencia, es un lugar terapéutico, educativo. ... COmunicar quiere decir ofrecer la cogestión de la enfermedad (de la salud), esto es, el acompañamiento profesional de la enfer-

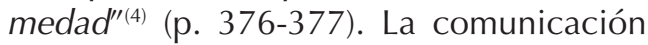
en salud puede ser comprendida a partir de cuatro componentes: 1) objetivos de los participantes en el encuentro; 2) necesidades, creencias, valores, conocimientos, habilidades y emociones de cada participante; 3) el proceso de comunicación y la forma como los mensajes son enviados y recibidos; y 4) la verificación del entorno en que la comunicación ocurre, tanto en el entorno más inmediato como en un contexto más amplio ${ }^{(5)}$. La verificación de estos cuatro componentes es importante a la hora de informar y comunicar en oncología.

Informar al paciente oncológico de su enfermedad conlleva aspectos positivos como respetar el derecho del paciente a estar informado y conocer su propia realidad. El enfermo tiene un nuevo motivo en su vida, luchar contra su enfermedad, por lo que colabora más en el tratamiento y se adapta mejor a la realidad. Para ello la información aportada tiene que ir cargada de esperanza, ese impulso que nos incita a seguir hacia delante. Vivir sin esperanza es empezar a morir, señala Flórez ${ }^{(6)}$. Pero informar también puede implicar aspectos más negativos, principalmente el hecho de que las personas de su entorno más cercano piensen que puede ser muy duro para el paciente y que es mejor protegerlo y ahorrarle las respuestas emocionales inmediatas. Sin embargo, debemos ser conscientes de que no decir la verdad al paciente no quiere decir que ésta no se conozca, lo cual puede ser bastante más contraproducente para el afectado y desencadenar problemas en su relación con el equipo sanitario y con su familia. Compartir la información con los familiares y con los profesionales permite al enfermo fortalecer su autoestima, sintiéndose parte activa en las decisiones que van a tomarse sobre su vida y contar con la posibilidad de expresar sus preocupaciones y temores. Por ello, en ningún momento del proceso podemos olvidar el destacado papel que juega la familia. A este respecto, Labrador y Bara $^{(7)}$ señalan que a pesar de que la mayoría de las personas desearían ser informadas en el caso de padecer un cáncer, su opinión cambia cuando es un familiar el afectado. Es muy frecuente que la familia intente que el paciente ignore que padece cáncer, ellos mismos no lo reconocen explícitamente y en muchos casos piensan que es mejor no hablar de ello. Su intención fundamental es proteger al paciente, creen que si no conoce el diagnóstico le ahorrarán malestar y sufrimiento.

No existe acuerdo acerca de la información que debe darse a una persona con cáncer sobre el diagnóstico, pronóstico y tratamiento de la enfermedad. Hasta no hace demasiado tiempo, en España lo más común era que el paciente no co- 
nociera con exactitud el diagnóstico y/o el pronóstico de su enfermedad. El médico informaba poco o lo hacía de manera ambigua y entre el personal sanitario y la familia existía un "pacto de silencio" que les hacía ocultar al enfermo gran parte de la información. Sin embargo, cada vez es más frecuente la tendencia a informar debido a imperativos legales, a la demanda de los propios enfermos y a que se ha pasado a un modelo de atención médica que otorga al paciente un papel más activo: el enfermo desea poder participar en las decisiones clínicas con respecto al proceso diagnóstico y a las propuestas terapéuticas. El problema está en cómo ejercer efectivamente esa participación en una enfermedad tan compleja como el cáncer ${ }^{(8)}$. Se ha pasado de un modelo biomédico a un modelo biopsicosocial, que defiende una visión multidimensional de la enfermedad como un hecho social, cultural y políticoeconómico $^{(9)}$. Este cambio de modelo también afecta a la relación médico-paciente en la que tiende a abandonarse el modelo paternalista para evolucionar hacia un modelo más deliberativo y participativo(8). Se ha constatado que los pacientes informados adecuadamente tienen una mejor adherencia a los tratamientos, son más proclives a la participación activa en el tratamiento y muestran una mejor adaptación psicológica y social a largo plazo $0^{(7,8,10,11)}$.

Kazimierczak et al. ${ }^{(12)}$ señalan que la información y el proceso de comunicación en el que se aporta, se llevan a cabo en un contexto sociocultural concreto y definido además por las personas directamente implicadas, que tienen sus propias necesidades y expectativas. Es una relación entre personas que pertenecen a dos culturas diferentes, la del profesional y la del paciente y su familia. Ambas tienen que aproximarse pues tienen un objetivo común que es la curación ${ }^{(4)}$. El médico es el especialista en la enfermedad y el que conoce los tratamientos, pero el enfermo es quien mejor conoce su cuerpo, sus necesidades y sus expectativas ${ }^{(3)}$. Pero esta aproximación no siempre se produce pues estamos frente a una relación en claro desequilibrio ya que el poder de tomar decisiones sobre la salud del afectado está en manos de una de las partes, la del médico. Si éste no es capaz de comunicarle la verdad del diagnóstico, de transmitir cercanía y confianza, la relación puede colapsarse con los consiguientes perjuicios para el enfermo. Los problemas también pueden aparecer del lado del enfermo si, por ejemplo, no reconoce su enfermedad.

El oncólogo debe transmitir una "verdad soportable" que debe ser el resultado de una "decisión médica compartida" que Peiró et al. ${ }^{(13)}$ definen como el "intento de los oncólogos de comunicarse y de compartir con los pacientes su experiencia de enfermedad". El doctor toma la decisión si no hay comunicación con el enfermo, cuando sí la hay ambos deben discutir y elegir juntos el mejor tratamiento. Nelson et al. ${ }^{(14)}$, en un estudio con pacientes con cáncer de pulmón, verificaron que los pacientes poco o nada hablaron con sus médicos sobre puntos importantes de su enfermedad y tratamiento, tales como síntomas, pronóstico, riesgos y beneficios potenciales del tratamiento, necesidades prácticas, cuidados hospitalarios y preocupaciones espirituales. Estos temas de preocupación son generalizables para pacientes con diferentes edades, estadio de la enfermedad y sexo, y son resultado de la falta de atención a las necesidades del paciente.

Con respecto a la decisión sobre el tratamiento, Brundage, Feldman-Stewart y Tishelman ${ }^{(5)}$ afirman que el proceso de decisión del paciente en oncología ocure con más calidad cuando el paciente consigue integrar el conocimiento basado en evidencias que el médico le informa y sus preferencias personales y valores sobre su salud y su vida. Existen herramientas 
de apoyo a la decisión (decision aids) que traducen las evidencias científicas existentes sobre el tratamiento de una forma que puede ser fácilmente comprensible para el paciente, ofreciéndole algunas informaciones y opciones de tratamiento, sus respectivos beneficios y sus riesgos. Para los autores, los médicos pueden ser entrenados para fortalecer su habilidad de comunicar, lo que puede tener un impacto práctico en la tomada de decisión sobre el tratamiento por parte del paciente. Cuando la información no es ofrecida de forma adecuada al paciente, o él entiende su tratamiento más basado en esperanzas que en hechos, las decisiones podrán ser equivocadas y no reflejarán sus verdaderos valores y deseos ${ }^{(15)}$.

El momento de la comunicación del diagnóstico también es fundamental y de cómo se haga, va a depender, en gran parte, la adaptación del enfermo a la situación. Aunque es un momento concreto en el que se le dice que tiene cáncer eso no significa que toda la información que pueda necesitar se aporte en ese momento, pues esto solo generaría confusión y ansiedad. La información debe ser secuencial y por tanto depender de las diferentes fases del proceso terapéutico y debe adaptarse a las necesidades del paciente, y a aquello que cada uno quiere saber. Lo difícil es conocer la realidad de cada enfermo. Además tiene que ir siempre acompañada de un claro mensaje de esperanza. Las primeras palabras que el enfermo recibe sobre su diagnóstico quedarán grabadas en su mente y son muy difíciles de borrar. Vidal et al. (3) plantean que el "diagnóstico es uno de los momentos de mayor tensión y más difícil emocionalmente, porque es dónde se confirma la sospecha y la persona se identifica con su papel de enfermo; implica una respuesta inicial, caracterizada por la incredulidad, el rechazo y el impacto de que la noticia pueda ser verdad, acompañada a menudo de un cierto sentimiento de "anestesia emocional", produciéndose un período de distanciamiento e introspección que dificulta el procesamiento de la información proporcionada" (p.30-31). Los autores señalan que aunque los pacientes comprendan adecuadamente el diagnóstico y las posibilidades de tratamiento, es muy posible que inmediatamente después de la consulta olviden parte de la misma.

Aunque la tarea de comunicar el diagnóstico es una tarea habitual, ello no le resta complejidad. En primer lugar es necesario tener en cuenta la información que posee el enfermo cuando llega al oncólogo, ya que según Peiró et al. ${ }^{(13)}$ solo el $20,1 \%$ de los afectados cuentan con información precisa sobre el diagnóstico cuando son derivados al servicio de oncología. A esta información se añadirá la aportada por el médico. La reacción inicial será de miedo y desconcierto, ya que es una enfermedad que todavía es habitual asociar a dolor y a duros tratamientos que conllevan un gran deterioro físico y psicológico, y que en muchos casos piensan que acabará con su vida. Ante esta situación, cuando se informa a los pacientes del diagnóstico, la mayoría se quedan bloqueados y aunque están escuchando, no procesan la información que se les está ofreciendo. Unos reaccionan Ilorando, otros enfadándose, a otros les confirma lo que sospechaban y algunos se quedan tan impactados que no dicen nada; reacciones todas ellas normales y de las que tiene que ser consciente la persona que está dando la información. Estas reacciones ante el diagnóstico y su afrontamiento varían dependiendo de aspectos como la conciencia que el enfermo tiene de la enfermedad, de su historia personal, del significado que de a la enfermedad, de las consecuencias de la misma y de los recursos de afrontamiento que puede poner en marcha ante esta situación ${ }^{(3)}$. Navarro et al. ${ }^{(16)}$ señalan que, en general, los pacientes dan más importancia a los aspectos relacionados con la competencia del médico y la información sobre la en- 
fermedad y los tratamientos que a los aspectos de apoyo emocional y contextual. Hack, et al. ${ }^{(17)}$ también corroboran que, en el caso de las mujeres con cáncer de mama, la mayor demanda inicial está relacionada con aspectos biomédicos, seguido de aspectos administrativos y emocionales.

Pero no sólo la comunicación del diagnóstico es tarea difícil, sino también la comunicación del pronóstico en oncología. Para Russel y Ward ${ }^{(15)}$, existen factores relacionados con los médicos y factores vinculados al paciente que dificultan el proceso de comunicación del pronóstico. Con respecto a los factores de los médicos, es posible señalar: poca confianza en su habilidad de pronosticar; tiempo insuficiente para ello; miedo de destruir la esperanza y de provocar estrés psicológico en el paciente; miedo de ser culpabilizado por el enfermo; miedo de confrontar sus propias emociones y su miedo a la muerte; sentimientos de inadecuación, presencia de burnout y sobrevaloración del conocimiento del paciente. Por otro lado, los factores vinculados al paciente son: ambivalencia en saber su pronóstico; negación, preferencia por dejar los detalles del tratamiento a otra persona; miedo de ofender al médico con sus preguntas y de hacerle perder su tiempo; miedo de perder la esperanza y dificultad en entender los términos técnicos de la medicina. Los autores señalan que a pesar de las dificultades el conocimiento del pronóstico es importante para que el paciente pueda tomar decisiones importantes sobre su vida, y también sobre su muerte, preparándose para lo que tiene que afrontar en el futuro. Las consecuencias que se derivan de un proceso de comunicación inadecuado afectan a la adaptación a la enfermedad y a la calidad de vida: ansiedad, depresión, desesperanza, sufrimiento, rechazo de las medidas terapéuticas y uso de medidas alternati$v s^{(16)}$. La información es una herramienta fundamental para el paciente oncológico pues facilita su participación en la gestión de sus cuidados de salud, el autocuidado y en la toma de decisiones ${ }^{(12,18)}$.

\section{EL PROCESO DE COMUNICACIÓN CON EL ENFERMO EN TRATAMIENTO DE QUIMIOTERAPIA}

La comunicación es indispensable para conseguir una atención humanizada ya que permite generar un contexto adecuado en el que se aporte una atención humana y cercana para que el cuidado y la curación se lleven a cabo de la mejor manera posible. Los profesionales deben ser capaces de transmitir seguridad, confianza y respeto. Sentirse escuchado contribuye de forma significativa a valorar positivamente la información recibida y contribuye al cumplimiento terapéutico.

La comunicación con el enfermo oncológico es un proceso que debe comenzar cuando la persona consulta a un médico por alguna sintomatología que padezca y éste le aconseja realizar algunas pruebas (biopsia, escáner...) que pueden hacerle sospechar la posibilidad de encontrar "algo malo". La percepción de estar en riesgo para alguna enfermedad grave, como es el cáncer, incluye preocupación sobre la posible enfermedad, percepción de gravedad y probabilidad de tener la enfermedad ${ }^{(19)}$, y muestra que el proceso de auto-regulación de la persona está desequilibrado. Para resolver el problema, la persona busca ayuda médica que investigue los síntomas. El tiempo que pasa hasta la confirmación del diagnóstico y los días posteriores a éste hasta que comienza el tratamiento son descritos por la mayor parte de los pacientes como los más angustiosos. Es el tiempo de mayor ansiedad pues aún no han empezado a hacer nada para luchar contra la enfermedad. Si se confirma el diagnóstico, el proceso continúa durante un prolongado período de tiempo hasta que se produce 
una remisión de la enfermedad, ésta pasa a ser crónica o la persona fallece.

Como parte del Plan de Humanización que desarrollamos en la sala de quimioterapia del Hospital de la Santísima Trinidad de Salamanca realizamos una historia biopsicosocial de cada paciente y de su acompañante principal en la que recogemos información sobre el afrontamiento de la enfermedad, la comunicación del diagnóstico y la información que ha recibido sobre el mismo, el tratamiento y los efectos secundarios aparecidos, la estancia en la sala de tratamientos, la relación con el equipo sanitario y las posibles repercusiones en aspectos sociales como las relaciones familiares, la vida laboral o las relaciones sociales. La relevancia de los resultados obtenidos sobre la comunicación del diagnóstico y la información que reciben sobre la enfermedad, nos lleva a marcarnos como objetivo prioritario conseguir una comunicación que se adapte a las necesidades físicas y psicosociales de cada paciente, diseñando una información personalizada y conforme a las creencias de cada uno. No es informar por informar, es aportar una información precisa, estructurada y conforme a la historia clínica y psicosocial del paciente y del tipo de cáncer que padece. Esta información debe tener como eje central la premisa que dirige nuestro Plan de Humanización Cada paciente es único y única es su enfermedad. En este apartado recogemos nuestra experiencia en la comunicación con pacientes oncológicos en tratamiento de quimioterapia.

No todos los pacientes desean ser informados, ni todos necesitan la misma información, ni en el mismo periodo de tiempo. En este proceso de comunicación con el enfermo, tan importante es hablarle como escucharle y tan importante es la comunicación verbal como la no-verbal. La información aportada tiene que permitir al enfermo que se sienta parte activa en el control y tratamiento de su enfermedad y encontrarse emocionalmente lo mejor posible. Como señalan Arrighi et al. (8) la mayor parte de los estudios recientes plantean que los enfermos de cáncer desean recibir mayor información, pero la cantidad y calidad que debe ser aportada, cómo esta es utilizada y cómo influye en la toma de decisiones terapéuticas, son aspectos que aún deben precisar las investigaciones al respecto. Además no debe olvidarse nunca que existen pacientes que no quieren involucrarse en las decisiones respecto a los tratamientos a seguir y que esta es también una opción que debe respetarse.

La influencia del equipo sanitario es fundamental durante todo el tiempo que están en tratamiento. En el hospital en el que trabajamos, los enfermos no disponen de atención integral por equipos interdisciplinares y multiprofesionales como ocurre en otros hospitales. Normalmente el cáncer es diagnosticado por el oncólogo que, en función del tipo de cáncer y del estadio en el que se encuentra, además de otras variables, establece el protocolo de tratamiento a seguir. El desarrollo y evolución de la enfermedad son controlados por el oncólogo antes del inicio de cada ciclo lo que motiva que exista un contacto directo y continuo con el enfermo y con la persona que le acompaña a las consultas, normalmente un familiar directo.

El espacio físico en el que trabajamos es una habitación rectangular en la que se puede atender a un máximo de ocho pacientes en el turno de la tarde, que pueden estar acompañados por la persona que deseen todo el tiempo que dura el tratamiento. La sala está ocupada durante todas las mañanas de lunes a viernes y los lunes por la tarde. Las reflexiones que a continuación presentamos están basadas en el trabajo con los enfermos que acuden al turno de tarde. El equipo profesional que les atiende está integrado por oncólogo, secretaria, enfermera de oncología y psi- 
cóloga (siempre son las mismas personas excepto en los periodos vacacionales).

El paciente es derivado a la sala por el oncólogo. El primer contacto con la enfermera y la psicóloga se produce o bien al terminar la consulta en la que se le comunica que tiene que iniciar tratamiento de quimioterapia o bien en el momento que acude a recibir su primer ciclo. La decisión depende del propio paciente.

Cuando llega por primera vez la sala de tratamientos, el paciente ya conoce el diagnóstico, que le ha sido comunica$\mathrm{do}^{7}$, en la mayor parte de los casos, por un médico, normalmente el oncólogo, el patólogo o el cirujano, y en un pequeño porcentaje por algún familiar. Al llegar, la enfermera y la psicóloga le reciben, se presentan y les indican dónde pueden sentarse y cómo va a realizarse el tratamiento. Una vez que ya está conectado al equipo a través del cual se le aplicará la medicación y que el resto de los pacientes también lo están, ambas se sientan junto al nuevo paciente y su acompañante y les hablan sobre la enfermedad, el tratamiento, los efectos secundarios que pueden aparecer $y$ en caso de que surjan qué pueden hacer para mejorar su estado; a la vez, intentan ir resolviendo todas las dudas que van surgiendo en la conversación. Cualquier aspecto, tanto físico como emocional, que el oncólogo cree que es necesario considerar en el proceso de comunicación es comentado con la enfermera previamente a que el paciente inicie su primer ciclo. La información que se da a cada enfermo es personalizada y dosificada, atendiendo a las necesidades y estado físico y emocional de cada uno. Al ser el primer ciclo y dada la carga de ansiedad y miedo que el enfermo tiene, el objetivo fundamental de esta información es que tenga una visión

7 Desde enero de 2007 hasta la actualidad solamente una persona no conocía el diagnóstico verdadero al llegar a la sala, ni se le comunicó durante el tratamiento por exigencias de la familia. general del procedimiento que debe seguir en cada ciclo y de las repercusiones que puede tener. La información se completa con la entrega del libro Manual para el paciente oncológico y su familia ${ }^{8}$ y de varios folletos de la Asociación Española contra el Cáncer. Con el objetivo de que el paciente no se sienta abrumado por la información, comentamos el contenido del libro con el enfermo y su acompañante centrándonos en los aspectos que consideramos más significativos para su caso concreto. En este sentido, les damos marcadas las páginas que, en principio, puede esperarse que sean más importantes en cada caso. A partir de este primer ciclo el proceso de comunicación sobre la enfermedad continua trabajándose en la sala en cada ciclo y en las consultas de control de cada paciente con su oncólogo, inmediatamente antes de cada ciclo. El contacto directo y continuo con los afectados y sus familiares nos aportan información sobre cómo se ha realizado el proceso de comunicación en lo que respecta a la información que se ha dado al paciente sobre el diagnóstico, cómo se ha realizado este proceso y cómo el paciente y su familia han vivido y sentido ese tiempo inicial de la enfermedad. Esta información es fundamental para poder subsanar posibles errores y malentendidos con respecto a la enfermedad y su desarrollo, que pueden incidir negativamente en el proceso de comunicación.

Un aspecto clave para conseguir un adecuado proceso de comunicación está basado en la relación que se establezca entre el equipo de profesionales y el enfermo y su familia. En nuestro caso, la valoración de dicha relación por parte de los

8 De Cáceres, $M^{\mathrm{a}}$ L.; Ruiz, F.; Germá, J.R. y Busques, C. (2007). Manual para el paciente oncológico y su familia. Barcelona: PfizerOncología. En fechas muy recientes el libro ha dejado de publicarse en papel por lo que, siempre que es oportuno, les decimos el enlace web en el que pueden descargarlo y/o les entregamos impresas las partes necesarias para cada caso. 
pacientes y sus familias, es altamente positiva. Establecen una relación de confianza que les aporta tranquilidad y seguridad. La enfermera de oncología encargada de aplicarles los ciclos es siempre la misma persona, salvo, como ya hemos señalado, en los periodos vacacionales y la relación con ella es decisiva en la adhesión del paciente al tratamiento. Ven en ella una persona a la que siempre pueden recurrir, que les escucha y atiende, que intenta resolver sus dudas, les ayuda a hacer frente a sus temores y les aconseja cómo paliar en lo posible los efectos secundarios de los tratamientos. La presencia en la sala de la psicóloga también es valorada positivamente por los pacientes y sus familias. Les atiende a la llegada a la sala y les indica dónde pueden sentarse, para evitar interacciones negativas entre algunos pacientes; por ejemplo, sentar a un paciente que no quiere hablar sobre su enfermedad al lado de otro que no para de contar su propia experiencia. Pasa toda la tarde en la sala, charlando con todos a la vez, o manejando situaciones emocionales concretas que surgen en el día a día y que afectan individualmente a algún paciente. A media tarde compartimos un café o una infusión y un dulce que siempre de manera espontánea lleva alguno de los afectados. A lo largo de la tarde hablamos no solo de aspectos relacionados con la enfermedad sino de cualquier otro tema que no tenga nada que ver con el cáncer. Compartimos experiencias, bromas, cotilleos ... todo ello procurando crear un ambiente distendido, cordial, en el que se sientan queridos y que les proporcione tranquilidad y seguridad. Nuestro objetivo es mimar a los pacientes y por ello nuestro proyecto de intervención se denomina MIMOS. Cuando detectamos que un paciente tiene algún problema psicológico que no es posible atender debidamente en la sala se le plantea la posibilidad de acudir a la AECC (Asociación Española Contra el
Cáncer). Nosotras mismas hablamos con las profesionales de la Asociación para derivarles el caso.

Trabajamos la aceptación del diagnóstico individualmente con cada paciente. Como ya hemos señalado, salvo contadas excepciones, cuando el enfermo llega a la sala para el primer ciclo ya conoce el diagnóstico verdadero. El tiempo desde que lo conoce varía, principalmente dependiendo de si se ha realizado intervención quirúrgica, en cuyo caso, la quimioterapia no empieza hasta que el paciente no se ha recuperado. De todos modos, en la mayor parte de los casos, este tiempo no ha sido suficiente para que el paciente asuma la situación, a lo que se une enfrentarse al miedo específico a la quimioterapia y las leyendas que giran alrededor de sus efectos secundarios. Por ello, nuestro primer cometido es tranquilizar al enfermo aportándole toda la información que precise sobre la enfermedad y el tratamiento a seguir desde ese momento. En la actualidad estamos trabajando en el diseño de un modelo general de información escrita, un documento informativo, con aspectos explicativos comunes pero que se pueda individualizar para cada persona, pues cada uno necesita la información en un momento determinado y no todos demandan la misma información. Este documento no puede ser cerrado, pues además de que cada enfermo necesita un tipo de información, la demanda en un momento temporal distinto, por lo que necesitamos elaborar un formato de fichas que permita ir incorporando piezas en función de las necesidades, que varían dependiendo de numerosas variables, como la edad y el sexo. Por ejemplo, es diferente la información que demanda una mujer joven, casada y con hijos pequeños, a una mujer de edad avanzada con hijos adultos.

En el hospital en el que trabajamos diferenciamos cuatro etapas en el proceso de información y comunicación con nuestros 
pacientes. En primer lugar el tiempo previo al diagnóstico, a continuación el momento en el que se confirma el diagnóstico y se le cuenta al enfermo y en tercer lugar todo el proceso de comunicación sobre el desarrollo de la enfermedad desde ese momento. A estas fases les sigue una última que será diferente si el paciente pasa a ser un superviviente o si el pronóstico es negativo y hay altas posibilidades de fallecimiento. En cada una de estas etapas es necesario que atendamos a numerosas variables.

\section{A) Primera etapa: Tiempo previo al diag- nóstico}

En este periodo incluimos todo el tiempo previo al diagnóstico, desde que el posible enfermo comienza a consultar al médico porque no se encuentra bien. En estos días iniciales la persona se somete a numerosas pruebas diagnósticas y aún puede que se encuentre relativamente tranquila. Esta tranquilidad se va perdiendo a medida que la persona es derivada a distintos especialistas. Este tiempo pasa a estar marcado por la incertidumbre y el miedo, que derivan en una gran carga de ansiedad para el "posible enfermo" y su familia. Es un tiempo en el que la persona no tiene ningún tipo de asistencia ni apoyo psicológico a pesar de lo necesario que sería trabajar durante estos días, tanto si posteriormente se confirma el diagnóstico como si no.

B) Segunda etapa: Información del diagnóstico y de los posibles tratamientos a seguir

Cuando los resultados de las pruebas confirman a la persona como enferma de cáncer pasamos a la siguiente etapa en la que se informa al ya enfermo de la nueva situación. Hay que contarle qué es lo que le sucede y a qué tratamientos puede optar para recuperarse. La mayor parte de las ve- ces esta información se la da el oncólogo al que ha terminado derivado y que será el encargado de dirigir el tratamiento y de llevar el control de la enfermedad desde ese momento. Se intenta que la información sobre el diagnóstico se le comunique al paciente con cariño y amabilidad, normalmente en el despacho del médico y acompañado por un familiar. Hay más quejas en este aspecto cuando el diagnóstico se ha dado por otro profesional y/o en otros hospitales. Este descontento es más frecuente en los acompañantes que en los propios enfermos. Normalmente porque en estos casos son los familiares quienes han recibido en primer lugar el diagnóstico, comunicado bruscamente, y es después, cuando hablan con el propio afectado y con su acompañante, cuando la información se ofrece con más cuidado y amabilidad.

Es importante destacar que tanto el paciente como el acompañante, opinan que no todos los enfermos necesitan el mismo tipo de información ni en el mismo momento, que cada uno tiene unas necesidades que deben tenerse en cuenta. Pero, en cualquier caso, consideran que es fundamental y prioritario estar bien informado.

La información debe dosificarse, quieren saber qué les ocurre y qué les va a suceder en su vida desde ese momento en adelante, pero tienen dificultades para asumir en una sola consulta la cantidad de información que se les puede aportar. Es difícil comprender en los minutos que dura la consulta, que el médico confirme que tienes cáncer y que te van a someter a tratamiento de quimioterapia, de radioterapia o que necesitan una intervención quirúrgica. Por muchas sospechas que el enfermo y su familia tuvieran al respecto, en el momento de recibir la confirmación las personas sienten miedo y angustia, desconocen qué va a pasar con su vida y les resulta difícil procesar la información que están recibiendo. 
C) Tercera etapa: Proceso de comunicación equipo sanitario-paciente-familia

A partir de aquí comienza la etapa que vamos a denominar "de comunicación" y que es la más duradera puesto que finaliza con la recuperación o el fallecimiento del paciente.

Una vez confirmado el diagnóstico la búsqueda de información sobre la enfermedad y los posibles tratamientos se convierte en una estrategia de afrontamiento habitual, tanto por parte del afectado como de los familiares y de forma especial cuanto más jóvenes son.

Las tres vías más frecuentes para buscar información adicional son, en primer lugar, Internet, en segundo lugar hablar con familiares y amigos con profesiones sanitarias y por último consultar en libros y revistas. Sobre el diagnóstico, si existen familiares o amigos sanitarios, lo primero que hacen es hablar con ellos, incluso si es posible les acompañan a la consulta con el oncólogo.

A mayor edad los enfermos demandan menos información, tanto sobre el diagnóstico como sobre el desarrollo posterior de la enfermedad. El afrontamiento de la enfermedad está más vinculado a la resignación y por lo tanto consideran que es suficiente la información que se les da. Adoptan un papel más pasivo y acorde con el modelo biomédico que dejaba en poder del médico toda la responsabilidad sobre la enfermedad y el enfermo asumía sin ningún cuestionamiento todo lo que se le planteaba.

Tanto los afectados como los acompañantes señalan el destacado papel que juega la familia durante todo el proceso de información y comunicación. La familia posee información, en muchos casos más precisa que el enfermo y antes de que él la conozca. Aunque el diagnóstico lo sepan ambos, el pronóstico, cuando es negativo, suele conocerlo en primer lugar la familia.
Enfermos, familiares y amigos necesitan ayuda para hablar sobre la enfermedad sin intentar engañarse mutuamente. El paciente tiene que ser capaz de expresar sus sentimientos, sus miedos, sus angustias, sus preguntas, necesita poder hablar con su familia del presente pero también del futuro y, sobre todo, de cómo reajustarse a la nueva vida surgida después del diagnóstico. La familia necesita incorporar el diagnóstico y todo lo que éste conlleva (consultas médicas, pruebas, tratamientos) en su vida cotidiana y realizar los ajustes necesarios. Para conseguirlo ambas partes tienen que ser capaces de expresar sentimientos, necesidades y demandas reales, sin intentar continuamente proteger al otro para que no sufra.

La sala de tratamientos de quimioterapia en la que trabajamos es el espacio mejor para trabajar esta comunicación debido a varias razones:

- La cantidad de tiempo que pasan en dicha sala con nosotros. Tanto en cada ciclo individual (la duración media podría establecerse en dos horas) como en la duración global del tratamiento.

- La estrecha relación que se crea entre los enfermos y sus familiares con los profesionales que les atienden en la sala.

- La relación que se establece con los otros pacientes y familiares con quienes se habla habitualmente sobre la enfermedad.

Con respecto a la gestión de la información en la sala no podemos presentar un patrón común y mayoritario sino que tenemos que hablar de un continuum que va desde las personas que no quieren hablar nada sobre la enfermedad a los que continuamente quieren plantear conversaciones relacionadas con su situación. Dependerá de los pacientes que cada día reciban el 
ciclo y de cómo se encuentren ese día. Sí podemos señalar una tendencia común que indica que no tienen inconveniente en hablar sobre la enfermedad, aunque les molestan los pacientes que tienen su enfermedad como único tema de conversación. Pero, como ya hemos señalado, también hay pacientes que no quieren hablar sobre el tema, por ello, uno de los aspectos que trabajamos especialmente en el Plan de Humanización es el control de la comunicación que surge cada día mientras están recibiendo el ciclo: información sobre los efectos secundarios, sobre las pruebas que les realizan y/o los resultados de sus marcadores. Es el momento en el que los pacientes más información intercambian pues cada uno cuenta qué efectos secundarios han aparecido desde el último ciclo y cómo han reaccionado a ellos. Además de plantear cuestiones sobre las siguientes repercusiones que pueden aparecer.

D) Cuarta etapa: el paciente pasa a ser un superviviente o fallece

Esta última etapa evidentemente es muy diferente dependiendo del curso de la enfermedad:

- Si el enfermo se convierte en un superviviente. La eficacia de los tratamientos actuales y el trabajo relacionado con la prevención y el diagnóstico precoz, motivan que el cáncer se cure cada vez en mayor número de afectados. Esto deriva en una clara necesidad de comunicación e información sobre lo que ocurre después del cáncer a nivel tanto físico como psicológico y social y en todas las esferas de la vida, en el trabajo, en las relaciones familiares y de pareja, en las relaciones sociales. En este caso como demuestran numerosos estudios actuales ${ }^{(20)}$, nos encontramos frente a una etapa clave y poco trabajada desde los diferentes profesionales que atienden al enfermo oncológico.

Dada la importancia que pensamos que tiene la necesidad de atender al superviviente nuestras investigaciones futuras estarán encaminadas en este sentido, pues hipotetizamos que la sala de tratamientos puede convertirse en el marco ideal para una consulta de enfermería y psicología a la que puedan acudir para resolver todas sus dudas y para realizar una adecuado seguimiento de su situación.

- Si el pronóstico de la enfermedad empeora, el paciente y su familia inician una fase final especialmente complicada en cuanto al proceso de comunicación se refiere ya que los aspectos a controlar incluyen el tratamiento del dolor, información sobre la muerte y cómo quieren vivir los momentos finales. Esta etapa queda al margen de la sala de tratamientos por lo que no forma parte del Plan de Humanización y además excede los objetivos de este artículo. Sin embargo, el estrecho contacto que se establece entre los profesionales y los enfermos y sus familiares motiva que también les acompañemos en estos últimos momentos.

\section{SUGERENCIAS Y REQUISITOS NECESARIOS PARA UNA COMUNICACIÓN EFICAZ}

Para conseguir una comunicación eficaz deben tenerse en cuenta los siguientes $\operatorname{aspectos}^{(11,15,21-26)}$ :

- Es necesario conseguir empatizar con el paciente, entendiendo la empatía como la disposición de una persona a ponerse en la situación 
existencial de otra, a comprender su estado emocional, a tomar conciencia de sus sentimientos, a conocer su experiencia y asumir su situación $^{(23)}$. Pero a la vez hay que ser capaces de mantener la necesaria distancia terapéutica con el paciente. Para ello debemos evitar cometer tres errores frecuentes:

- Quitarle importancia a la situación diciendo al enfermo "no te preocupes".

- Contra argumentar; el paciente lo vive como nos lo cuenta por lo que tenemos que explicarle las cosas, pero no decirle "que va, eso no es así".

- Dar consejos y opiniones prematuros y no solicitados: el propio paciente debe marcar el ritmo de lo que hay que decirle.

- No olvidar la importancia de la comunicación no verbal, que por sí sola es una fuente de información para el enfermo, y que debe ser coherente con la comunicación verbal.

- Realizar una escucha activa: oír no es lo mismo que escuchar, escuchar implica mucho más.

- El profesional debe dar al enfermo la oportunidad de preguntar, para lo cual es necesario que consiga crear un clima de confianza y tranquilidad en el que el paciente y su entorno puedan expresar sus dudas $y$ plantear sus interrogantes con tranquilidad.

- Es necesario individualizar: cada paciente es único y única es su enfermedad y todo lo que ella conlleva.

- Debe facilitarse la expresión de emociones: es necesario que se permita y facilite al enfermo y a sus familiares que manifiesten sus sentimientos.

- Es indispensable el refuerzo de las conductas positivas del paciente $y$ de la familia, pues así mejora su autoestima y la relación con los profesionales. Para la familia es un momento muy angustioso por lo que si alguien les dice que lo están haciendo muy bien, se sienten útiles y estimulados.

- Es mejor sugerir que imponer: si el asunto es importante, debemos explicar porqué lo es y con eso seremos suficientemente convincentes.

- No podemos juzgar: nos encontramos ante una situación vital muy difícil y es necesario pensar que las reacciones no son a veces las más esperadas, y hay que intentar comprenderlas.

- No podemos mentir, lo cual tampoco quiere decir que sea oportuno y necesario contar toda la verdad de cualquier modo y en cualquier momento.

Todo ello con el objetivo de conseguir una comunicación saludable, "que se adapte específicamente a las necesidades psicosociales del paciente (concreto) que sufre una grave enfermedad y que va a tener repercusiones muy importantes de cara a su recuperación y al cuidado de la integridad y dignidad personales ${ }^{\prime \prime(6)}$ (p. 45).

Como reflexión final señalar que nuestro trabajo diario con las personas que están en tratamiento de quimioterapia nos lleva a plantear la comunicación como una tarea fundamental para el profesional sanitario, y a señalar la necesidad de conseguir establecer una comunicación saludable con el paciente y sus familiares como un claro signo de una buena práctica clínica. Una buena comunicación e información facilitan la adaptación y la percepción de control por parte del paciente, le ayudan a vivir el día a día, a conservar su autonomía, a reducir el nivel de ansiedad y a marcarse objetivos realizables. 


\section{REFERENCIAS BIBLIOGRÁFICAS}

1. Sociedad Española de Oncología Médica (SEOM) El cáncer en España 2014. En línea. [Acceso 1 de febrero de 2014]. Disponible en: http://www.seom.org/es/ prensa/el-cancer-en-espanyacom/104582el-cancer-en-espana-2014

2. Asociación Española Contra el Cáncer (AECC) En líneas [Acceso 15 de febrero de 2014]. 2013. Disponible en: https:// www.aecc.es/SobreEICancer/Paginas/ Sobreelc\%C3\%A1ncer.aspx

3. Vidal $M^{a} L$, Sánchez $P$, Corbellas $C$, Peiró $\mathrm{G}$, Godes $\mathrm{M}$, Blasco $\mathrm{A}$, et al. Estudio descriptivo sobre el recuerdo del paciente tras la comunicación del diagnóstico y del pronóstico en oncología. Psicooncología 2007;1:21-33.

4. Boixareu RMa . La relación terapéutica. La humanización de la técnica. En: Boixareu $\mathrm{RM}^{\mathrm{a}}$. De la antropología filosófica a la antropología de la salud. Barcelona: Herder, 2008, p.375-84.

5. Brundage MD, Feldman-Stewart D, Tishelman C. How do interventions designed to improve provider-patient communication work? Illustrative application of a framework for communication. Acta Oncol 2010;49:136-43. Doi: 10.3109/02841860903483684

6. Flórez JA. La comunicación y comprensión del enfermo oncológico. Madrid: Ediciones Ergón, 1997.

7. Labrador FJ, Bara E. La información del diagnóstico a pacientes de cáncer. Psicooncología 2004;1(1):51-66.

8. Arrighi E, Jovell AJ, Navarro $M^{\mathrm{a}} \mathrm{D}$. El valor terapéutico en oncología. La perspectiva de pacientes, familiares y profesionales. Psicooncología 2010;7:363-74.

9. Martínez Hernáez A. Cultura, enfermedad y conocimiento médico. La antropología médica frente al determinismo biológico. In: Esteban ML, editor. Introducción a la Antropología de la Salud. Bilbao: OSALDE, 2007, p. 11-43.
10. Holland J, Lewis S. La cara humana del cáncer. Barcelona: Herder, 2003.

11. Gómez Sancho M. Cómo dar las malas noticias en Medicina. Madrid: Arán, 2006.

12. Kazimierczak CA, Skea ZC, DixonWoods M, Entwistle VA, Fedman-Steward D, N’Dow J, et al. Provision of cáncer information as a "support for navigating the knowledge landscape": Findings form a critical interpretative literature synthesis. Eur J Oncol Nurs 17:360-9; Doi: 10.1016/j. ejon.2012.10.002

13. Peiró G, Corbellas C, Camps C, Sánchez PT, Godes M, D'Ambra M, et al. Estudio descriptivo sobre el proceso de comunicar el diagnóstico y el pronóstico en oncología. Psicooncología 2006;3:9-17.

14. Nelson JE, Gay EB, Berman AR, Powell CA. Patients rate physician communication about lung cancer. Cancer 2011;15:521220. Doi: 10.1002/cncr.26152

15. Russell B, Ward A. Deciding what information is necessary: Do patients with advanced cancer want to know all the detais? Cancer Manag Res 2011;3:191-9. Doi: 10.2147/CMR.S12998

16. Maté J, Navarro E, Limonero JT, GómezRomero, MJ. Necesidades de comunicación e información en el paciente oncológico superviviente. Psicooncología 2010;1:127-41.

17. Hack THF, Pickles T, Ruether JD, Weir L, Bultz BD, Degner LF. Behind closed doors: Systematic analysis of breast cancer consultation communication and predictors of satisfaction with communication. Psychooncology 2010;19:626-36. Doi: 10.1002/pon.1592

18. Kern E, Moro L. Factores psicosociales relacionados con el autocuidado en la prevención, tratamiento y postratamiento del cáncer de mama. Psicooncología 2012;9:453-465. Doi: 10.5209/rev_ PSIC.2013.v9.n2-3.40916

19. Shiloh $\mathrm{S}$, Wade $\mathrm{CH}$, Roberts JS, Alford $\mathrm{SH}$, Biesecker B. Associations between risk perceptions and worry 
about common disease: A betweenand-within-subjects examination. Psychol Health 2013; 28:434-49. Doi: 10.1080/08870446.2012.737464

20. Ferro T, Prades J. Necesidades de información en el cáncer de mama y de atención en la supervivencia. Federación Española de cáncer de mama 2013. En línea. [Acceso 12/12/2013] Disponible en: http://fecma.vinagrero.es/documentos/estudio.pdf

21. Moro L. El enfermo de cáncer y su entorno. Estudio de una muestra psicosocial de pacientes con leucemias y linfomas. Barcelona: Fundación La Caixa, 1994.

22. Moro L. Variables que influyen en la respuesta psicológica al cáncer. Iberpsicología. Revista Electrónica de la Federación Española de Asociaciones de Psicología 1997;2. [Acceso 15 de diciembre de 2013]
Disponible en: http://www.fedap.es/ IberPsicologia/iberpsi2-1/moro/moro.htm

23. Bermejo JC. Apuntes de Relación de ayuda. Santander: Sal Térrea, 1998.

24. García-Cerón MA, Avarado-Aguilar S. La comunicación en la relación médico-paciente: perspectiva del paciente oncológico. Gamo 2009;8:119-126.

25. MacLean $M$, Cleland JA, Worrell $M$, Vögele C. What am I going to say here? The experiences of doctors and nurses communicating with patients in a cancer unit. Front Psychol 2:339. Doi: 10.3389/ fpsyg.2011.00339

26. McNair AG, Brookes ST, Davis CK, Argylopoulos M, Blazeby JM. Communication the results of randomized clinical trials: Do patients understand multidimensional patientreport outcomes? J Clin Oncol 2010;28:73843. Doi: 10.1200/JCO.2009.23.9111 